\title{
Three\#dimensional Magnetohydrodynamic Simulations of Radiatively Inefficient Accretion Flows
}

\section{Citation}

Igumenshchev, Igor V., Ramesh Narayan, and Marek A. Abramowicz. 2003. "Three\#dimensional Magnetohydrodynamic Simulations of Radiatively Inefficient Accretion Flows." The Astrophysical Journal 592 (2): 1042-59. https://doi.org/10.1086/375769.

\section{Permanent link}

http://nrs.harvard.edu/urn-3:HUL.InstRepos:41384952

\section{Terms of Use}

This article was downloaded from Harvard University's DASH repository, and is made available under the terms and conditions applicable to Other Posted Material, as set forth at http:// nrs.harvard.edu/urn-3:HUL.InstRepos:dash.current.terms-of-use\#LAA

\section{Share Your Story}

The Harvard community has made this article openly available. Please share how this access benefits you. Submit a story. 
Submitted to The Astrophysical Journal

\title{
THREE-DIMENSIONAL MHD SIMULATIONS OF RADIATIVELY INEFFICIENT ACCRETION FLOWS
}

\author{
Igor V. Igumenshchev \\ Laboratory for Laser Energetics, University of Rochester, 250 East River Road, Rochester, \\ NY14623; iigu@lle.rochester.edu \\ Ramesh Narayan \\ Department of Astronomy, Harvard-Smithsonian Center for Astrophysics, 60 Garden \\ Street, Cambridge, MA 02138; narayan@cfa.harvard.edu \\ Marek A. Abramowicz \\ Department of Astronomy and Astrophysics, Göteborg University and Chalmers University \\ of Technology, S-41296, Göteborg, Sweden; marek@fy.chalmers.se
}

\begin{abstract}
We present three-dimensional MHD simulations of rotating radiatively inefficient accretion flows onto black holes. We continuously inject magnetized matter into the computational domain near the outer boundary and run the calculations long enough for the resulting accretion flow to reach a quasi-steady state. We have studied two limiting cases for the geometry of the injected magnetic field: pure toroidal field and pure poloidal field. In the case of toroidal field injection, the accreting matter forms a nearly axisymmetric, geometrically-thick, turbulent accretion disk. The disk resembles in many respects the convection-dominated accretion flows found in previous numerical and analytical investigations of viscous hydrodynamic flows. Models with poloidal field injection evolve through two distinct phases. In an initial transient phase, the flow forms a relatively flattened, quasi-Keplerian disk with a hot corona and a bipolar outflow. However, when the flow later achieves steady state, it changes in character completely. The magnetized accreting gas becomes two-phase, with most of the volume being dominated by a strong dipolar magnetic field from which a thermal low-density wind flows out. Accretion occurs mainly via narrow slowly-rotating radial streams which 'diffuse' through the magnetic field with the help of magnetic reconnection events.
\end{abstract}


Subject headings: accretion, accretion disks — black hole physics — convection — MHD — turbulence

\section{INTRODUCTION}

This study is a continuation of our efforts during the last several years to understand the nature of underluminous accreting black holes. A class of radiatively inefficient solutions (Ichimaru 1977; Rees et al. 1982; Narayan \& Yi 1994, 1995a,b; Abramowicz et al. 1995; Narayan, Mahadevan \& Quataert 1998) has been influential in this field. A key feature of these solutions is that radiative energy losses are small so that most of the energy is advected with the gas. Narayan \& Yi (1994) came up with the name 'advection-dominated accretion' to describe such flows, and Lasota (1996) suggested the acronym ADAF (advectiondominated accretion flow). Usually by ADAFs one understands optically thin accretion flows with very low mass accretion rates, $\dot{M} \ll \dot{M}_{E d d}$, but ADAFs are also possible as optically thick flows with high accretion rates, $\dot{M}>\dot{M}_{E d d}$, where $\dot{M}_{E d d}$ is the Eddington accretion rate. In the later case, one uses the name 'thick accretion disk' (see e.g. Jaroszyński, Abramowicz \& Paczyński, 1980) or 'slim accretion disk' (Abramowicz et al., 1988) rather than ADAF.

Historically, ADAFs were initially studied within the framework of viscous hydrodynamics. In this approach, one assumes that the accreting gas has an 'anomalous viscosity' parameterized by the standard dimensionless viscosity parameter $\alpha$. Early analytic work revealed that the gas in an ADAF would be (i) gravitationally unbound because of a positive Bernoulli parameter, which might lead to a strong outflow (Narayan \& Yi 1994, 1995a), and (ii) convectively unstable (Gilham 1981; Begelman \& Meier 1982; Narayan \& Yi 1994, 1995a). The important role of these processes was not, however, realized until numerical two- and three-dimensional hydrodynamic simulations were carried out by a number of authors (Igumenshchev, Chen \& Abramowicz 1996; Igumenshchev \& Abramowicz 1999, 2000; Stone, Pringle \& Begelman 1999; Igumenshchev, Abramowicz \& Narayan 2000; McKinney \& Gammie 2002). These studies showed that the behavior of a viscous ADAF depends on the value of $\alpha$. If $\alpha$ is large, say $>0.3$, then there is a powerful bipolar outflow driven by the positive Bernoulli parameter. However, if $\alpha$ is small, say $<0.1$, the flow is dominated by convective motions which strongly modify the structure of the flow.

The convection-dominated low- $\alpha$ case was modeled analytically by Narayan, Igumenshchev \& Abramowicz (2000) and Quataert \& Gruzinov (2000), who coined the name 'convection-dominated accretion flow' (CDAF). Two key features of a CDAF are: (i) the Reynolds stress associated with the convective turbulence moves angular momentum inward 
rather than outward, and (ii) the density profile is much flatter than in the case of a pure ADAF $-\rho \sim R^{-1 / 2}$ rather than $R^{-3 / 2}$. The CDAF model provides a physical explanation for the low luminosity of accreting black holes, namely, the mass accretion rate is much reduced in the presence of convection.

Accretion models that are based on the principles of viscous hydrodynamics are limited by the fact that they need to invoke a mysterious 'anomalous viscosity.' Since the 'viscosity' in these models is very likely provided by magnetic fields via the magneto-rotational instability (Balbus \& Hawley 1991), a deeper understanding of accretion flows can clearly be obtained by analyzing the problem directly within the framework of MHD. Numerical MHD simulations of radiatively inefficient flows have been carried out recently by a number of authors (Hawley 2000, 2001; Stone \& Pringle 2001; Machida, Matsumoto \& Mineshige 2001; Hawley, Balbus \& Stone 2001; Hawley \& Balbus 2002; Casse \& Keppens 2002). However, the results obtained by the various authors have not been entirely consistent. For instance, some authors conclude that radiatively inefficient MHD flows behave like CDAFs (Machida et al. 2001), whereas others claim that the flows are very different (Hawley et al. 2001). It is not clear if the discrepancies arise from differences in the assumed geometry of the initial magnetic field or differences in the numerical techniques. In addition, magnetic stresses can have explicitly non-viscous behavior, such as causing angular momentum transport and accretion without any associated energy dissipation (Li 2003).

We present in this paper 3D numerical simulations that we have carried out of radiatively inefficient MHD accretion flows. In $\S 2$ we describe our numerical method and the initial and boundary conditions. In $\S 3$ we present numerical results for two topologies of the injected magnetic field: toroidal field ( $(3.1)$ and poloidal field (§3.2). In $\S 4$ we discuss the implications of our results. We argue that the toroidal field case has many points of similarity with the CDAF model (§4.1), while the poloidal field case behaves very differently ( $\$ 4.2)$. In fact, the steady state configuration that we obtain for the poloidal problem is unlike anything published previously in the literature; we suggest possible reasons for this (§4.3) and discuss the applicability of the obtained solutions to real accretion flows ( $\$ 4.4)$. In $\S 5$ we conclude with a summary. 


\section{SIMULATION TECHNIQUE}

\subsection{Magnetohydrodynamic Equations and Numerical Method}

We consider the dynamics of accreting plasma within the framework of the one-fluid MHD approximation. The relevant equations of resistive MHD take the following form:

$$
\begin{gathered}
\frac{d \rho}{d t}+\rho \nabla \cdot \mathbf{v}=0, \\
\rho \frac{d \mathbf{v}}{d t}=-\nabla\left(P_{g}+Q\right)-\rho \nabla \Phi+\frac{1}{4 \pi}(\nabla \times \mathbf{B}) \times \mathbf{B}, \\
\rho \frac{d \epsilon}{d t}=-\left(P_{g}+Q\right) \nabla \cdot \mathbf{v}+\frac{1}{4 \pi} \eta \mathbf{J}^{2}, \\
\frac{\partial \mathbf{B}}{\partial t}=\nabla \times(\mathbf{v} \times \mathbf{B}-\eta \mathbf{J}),
\end{gathered}
$$

where $\rho$ is the density, $\mathbf{v}$ is the velocity, $P_{g}$ is the gas pressure, $\Phi$ is the gravitational potential, $\mathbf{B}$ is the magnetic induction, $\epsilon$ is the specific internal energy, $\mathbf{J}=\nabla \times \mathbf{B}$ is the current density, $\eta$ is the resistivity, and $Q$ is the additional 'pressure' corresponding to a standard artificial viscosity.

We adopt the ideal gas equation of state,

$$
P_{g}=(\gamma-1) \rho \epsilon,
$$

with an adiabatic index $\gamma=5 / 3$, and we assume that there is no radiative cooling. We neglect self-gravity of the gas and take the gravitational potential to be entirely due to a central black hole of mass $M$. We employ a pseudo-Newtonian approximation (Paczyński \& Wiita 1980) for the potential:

$$
\Phi=-\frac{G M}{R-R_{g}}, \quad R_{g}=\frac{2 G M}{c^{2}},
$$

where $R$ measures the radial distance from the black hole and $R_{g}$ is the gravitational radius of the black hole.

Our numerical method is the MHD generalization of the hydrodynamic PPM algorithm (Colella \& Woodward 1984) and is identical to that used by Igumenshchev \& Narayan (2002). In this method the hydrodynamical part is solved by means of a variant of the Godunov method and the magnetic part is solved using a modified version of the method of characteristics (Stone \& Norman 1992). For reasons of numerical stability, we solve an equation for the internal energy rather than total energy. This requires use of the term $Q$ in 
equations (2) and (3), and the terms $\eta \mathbf{J}^{2} / 4 \pi$ and $\eta \mathbf{J}$ in equations (3) and (4), respectively. To avoid excessive loss of energy as a result of numerical reconnection of magnetic field lines, the resistivity is assumed to be non-zero and to have the following form (see Stone \& Pringle 1999),

$$
\eta=\eta_{0} \frac{|\nabla \times \mathbf{B}|}{\sqrt{4 \pi \rho}} \Delta^{2},
$$

where $\eta_{0}$ is a dimensionless parameter and $\Delta$ is the grid spacing.

The code operates on a three-dimensional nested Cartesian grid, which is designed so as to adequately resolve the large range of spatial scales spanned by the accretion flow. We designate the distance from the black hole to the face centers of the outermost cube of the grid as $R_{\text {out }}$. Igumenshchev \& Narayan (2002) discuss the code in more detail.

\subsection{Initial and Boundary Conditions}

The simulations are begun with non-magnetized 'empty space' surrounding the black hole; for technical reasons the empty space corresponds in actuality to a non-zero but extremely low density of matter. Mass is steadily injected into the computational domain inside a slender equatorial torus of thickness equal to about two grid-cells centered on the black hole and with a radius nearly equal to $R_{\text {out }}$. The injected matter has Keplerian rotation velocity and internal energy equal to a fraction 0.2 of the gravitational energy. The matter also has a magnetic field associated with it. We have adopted two configurations for the injected field: purely toroidal field and purely poloidal field (defined with respect to the equatorial plane of the torus).

In the toroidal injection case, the field is generated assuming that only the vertical component $A_{z}$ of the vector potential $\mathbf{A}$ is non-zero in the injected matter; the other two components, $A_{r}, A_{\phi}$, are set to zero. Here and below, we represent $\mathbf{A}$ in cylindrical coordinates $(r, \phi, z)$. In each time step, $A_{z}$ is increased within the torus as follows,

$$
A_{z}^{\text {new }}=A_{z}^{\text {old }}+\left(\frac{8 \pi \Delta \rho c_{s}^{2}}{\beta_{0}}\right)^{1 / 2} \Delta,
$$

where $\Delta \rho$ is the increase of the density in the torus due to the injection of matter, $c_{s}^{2}$ is the sound speed of the injected matter, and $\Delta$ is the grid spacing. The parameter $\beta_{0}$ measures the plasma $\beta$ of the injected material $\left(\beta \equiv P_{g} / P_{m}\right.$ where $P_{m}$ is the magnetic pressure). Since the injection of matter is axisymmetric, this model generates magnetic field with closed axisymmetric toroidal magnetic lines. In reality, however, the finite resolution of the Cartesian grid introduces small non-axisymmetric perturbations to the field structure. 
These act as seed perturbations in the simulations. Since $\mathbf{B}=\nabla \times \mathbf{A}$, simply increasing $\mathbf{A}$ does not necessarily lead to an increase of $\mathbf{B}$; at some moments of time $\mathbf{B}$ may actually be reduced by the procedure (8). Moreover, this procedure can break flux freezing of plasma in the injection region, by changing the topology of the field there. However, test simulations show that none of these effects is serious and the injected field does have the desired topology.

Magnetic field with poloidal topology is generated in a similar way as described above, with the same functional form given in equation (8), except that now only the component $A_{\phi}$ is taken to be non-zero, and $A_{r}$ and $A_{z}$ are both set to zero. In this case, the injected magnetic field lines take the form of poloidal loops around the torus.

In both the toroidal and poloidal cases, the presence of magnetic field in the injected material allows the redistribution of angular momentum via magnetic stresses. As a result, some of the injected matter loses its initial angular momentum and accretes onto the black hole, while the excess angular momentum is removed from the computational domain by the rest of the matter flowing out. With time, the initial inner 'empty space' becomes filled with accreting gas, and after several rotation periods as measured at the injection radius, a quasi-stationary accretion flow is established. We are mainly interested in the inner regions of the accretion flow, where the influence of the outer boundary and the injection region is not significant. Test simulations show that the dynamics and structure of the flow inside $\sim R_{\text {out }} / 2$ depend only weakly on the details of the injection region.

Absorbing boundary conditions are applied at both the inner and outer boundaries. The inner boundary consists of a sphere of radius $R_{i n}=2 R_{g}$ located inside the marginally stable orbit of the black hole, while the outer boundary corresponds to the outermost cube of the Cartesian grid at $R_{\text {out }}$. At both the inner and outer boundaries, matter is allowed to flow freely out of the computational domain, but no matter is allowed to return from outside. Magnetic field at both boundaries satisfies the divergence-free condition. The field at the inner boundary is defined assuming that $\mathbf{A}=\mathbf{A}_{\text {init }}=$ constant in the ghost cells located inside $R_{i n}$; here $\mathbf{A}_{\text {init }}$ is the initial (uniform) vector potential. This boundary condition on $\mathbf{A}$ introduces a jump in the magnetic field near $R_{i n}$. However, the jump has no effect on the inflowing matter because of the strong gravity and super-Alfvenic infall speed. At the outer boundary, the transverse components of the field with respect to the sides of the cube are assumed to be zero. This again introduces a jump in the field, which results in an artificial torque on the flow. The strength of this spurious torque increases with increasing field strength. 


\section{NUMERICAL RESULTS}

The simulations were done on a Cartesian numerical grid consisting of a number of nested subgrids of increasing resolution inward. The grid is designed to provide extra resolution closer to the black hole. The number of subgrids varies from 1 to 5 in different runs. Each subgrid consists of $64 \times 64 \times 64$ cells. The cell size in the innermost subgrid is $\Delta_{1}=0.5 R_{g}$, and each succeeding subgrid has its cell size increased by a factor of 2 . Therefore, in the simulations with 5 subgrids, the outermost subgrid has $\Delta_{5}=8 R_{g}$, and covers a cube of size $2 R_{\text {out }} \times 2 R_{\text {out }} \times 2 R_{\text {out }}$, where $R_{\text {out }}=256 R_{g}$. In practice, we use only a quarter of the full cubic domain, by focusing on a $90^{\circ}$ wedge around the $z$-axis. Thus, we employ $32 \times 32 \times 64$ cells along $x y z$ and use periodic boundary conditions in the azimuthal direction. The nested Cartesian grid has an important technical advantage with respect to a conventional spherical grid: there are no polar singularities, and therefore there is no reduction in the azimuthal grid-size near the pole. As a result, the time step defined by the Courant condition is significantly longer compared to a spherical grid, and for a given amount of computational effort we are able to run a simulation for a much longer time. On the other hand, angular momentum conservation is very good in spherical and cylindrical grids since the azimuthal equation of motion can be represented in an explicitly conservative form. A Cartesian grid does not have this advantage, so angular momentum is likely to diffuse more.

Spatial re-scaling $r \rightarrow r / R_{g}$ and time re-scaling $t \rightarrow t /\left(R_{g} / c\right)$ make the problem independent of the black hole mass $M$. Further, since we consider non-radiative flows, the density $\rho$, the pressure terms $P_{g}, Q$, and the electromagnetic terms $B^{2}, J^{2}$ in equations (1)-(4) are all proportional to the mass injection rate, $\dot{M}_{\text {inj }}$. Therefore, we may eliminate $\dot{M}_{\text {inj }}$ from the equations and consider only the scaled solutions.

When we run a time-dependent simulation using the above code, the system generally goes through an initial transient phase before relaxing to a steady state. Since we are mostly interested in the final steady state flow, we continue the calculations for a fairly long time - long enough that we feel from looking at the results that the model has reached a steady state. Even in steady state, the flow patterns are often time-dependent, so we need to consider the time-averaged properties of the flow. Typically, it takes about ten Keplerian rotational periods, as measured at $R_{\text {out }}$, to reach a steady-state, and we need to average over a few Keplerian periods in the final state to calculate its average properties.

All our models have been calculated assuming the resistivity parameter $\eta_{0}=0.3$. It has been found in previous MHD simulations (Igumenshchev \& Narayan 2002) that this value of $\eta_{0}$ is reasonable. On the one hand, $\eta_{0}$ is small enough that it does not excessively smooth out small-scale magnetic structures. At the same time it is large enough to prevent serious loss of energy through numerical field reconnection. 


\subsection{Simulations with Toroidal Injected Field}

The first set of simulations we describe involve the injection of matter with a toroidal field topology. These simulations were done with $A_{r}=A_{\phi}=0$ and with $A_{z}$ equal to the value given in equation (8). As explained in $\S 2.2$, this leads to a purely toroidal injected field. As the accreting matter moves in, however, poloidal field of either sign is generated locally, though the total magnetic flux through the equatorial plane is still zero to within the accuracy of truncation errors.

We have calculated a range of models with different values of the parameter $\beta_{0}$ (which describes the strength of the injected magnetic field) and with different numbers of nested Cartesian subgrids. We find that the flow pattern in steady-state is practically independent of $\beta_{0}$. The magnetic field in the inner regions of the flow reaches a saturation level which is almost the same for $\beta_{0}$ anywhere in the range 1 to $10^{4}$. The reason is that the magnetic field adjusts near the injection region itself to a certain saturation level, and maintains that level at all smaller radii. Machida et al. (2001) also found that the flow structure is independent of the initial strength of the toroidal magnetic field.

We discuss here our most advanced simulation, Model A, which was calculated on 5 subgrids with an outer radius $R_{\text {out }}=256 R_{g}$ and injected field parameter $\beta_{0}=10^{2}$. By the end of the simulation, after about ten orbits at $R_{\text {out }}$, Model A achieved steady state in a time-averaged sense. Figure 1 shows a snapshot of the density distribution in this model in meridional cross-section. We see that the accreting matter forms a thick disk, in which the density is concentrated at the equatorial plane and increases toward the black hole. The small-scale fluctuations of density which are visible are due to turbulent motions. The amplitude of the fluctuations is not large. Figure 2 shows a snapshot of the pressure distribution, which is seen to have even smaller amplitude fluctuations compared to the density. This is because the turbulence is subsonic.

The turbulent motions in the gas in Model A are generated by magnetic interactions, which provide the main dissipation mechanism. Most of the dissipation of energy arises from the resistive term, $\eta \mathbf{J}^{2} / 4 \pi$, in equation (3); the contribution from the artificial viscosity is very low since there are no shocks. During accretion, the binding energy of matter is efficiently converted into magnetic energy by two distinct mechanisms: field dragging by the radially converging flow (Shvartsman 1971), and field amplification by a magnetic dynamo associated with the magneto-convection and turbulence. In Fig. 3, we show a schematical example of how the local magnetic field may be amplified. A perturbed magnetic line is stretched in the azimuthal direction by the Keplerian differential rotation, following which the line self-reconnects, leaving behind a closed magnetic loop in addition to the original

line. This mechanism represents a non-linear stage of the magneto-rotational instability 
(Balbus \& Hawley 1991). Field amplification is accompanied by field dissipation via magnetic reconnection, which releases energy locally at the reconnection site. The combined effect of a large number of reconnection events, randomly distributed in time and over the disk volume, drives the turbulent motions and fluctuations in the flow.

One finds thus a feedback link between turbulence and reconnection: the turbulent motion tangles magnetic lines and amplifies the magnetic field, the tangled lines and turbulent motion increase the number of reconnection events, and the reconnections feed the turbulent motions through local dissipation of the field. Obviously, there is a saturation state in which the rate of amplification of magnetic energy is balanced by the rate of dissipation via reconnection.

In Fig. 4 we show a typical example of the topology of the magnetic lines as projected on the equatorial plane. Here and below, we use the line integral convolution method (LIC, Cabral \& Leedom 1993) to illustrate vector fields. We see that the lines are not simply stretched tangentially as we would expect for a pure Keplerian flow. Rather, the lines are strongly perturbed by the presence of many magnetic loops. In the meridional section shown in Fig. 5, we again see that reconnection and turbulent motion of matter result in a very complicated topology of poloidal magnetic lines. Especially in the equatorial regions, we see many magnetic vortices and loops of different spatial scales. In contrast, in the narrow polar regions, the magnetic topology is dominated by a regular field, in which magnetic lines are oppositely directed in the upper and lower funnels.

Figure 6 shows a snapshot of the plasma $\beta$ in meridional cross-section. We see that $\beta$ is highly inhomogeneous as a result of the stochastic reconnection and local dissipation of magnetic field described above. In individual fluctuations, the magnetic pressure can be up to $\sim 1-2$ orders of magnitude larger than the average value. However, the gas pressure $P_{g}$ fluctuates very little and $P_{m}$ never exceeds $P_{g}$. On average, $\beta \sim 10^{2}-10^{3}$ in the equatorial region of the accretion flow, and decreases towards the poles.

The dissipation of magnetic field via reconnection results in an increase in the entropy of the local gas. Figure 7 shows the distribution of the specific entropy in meridional crosssection. We see that the entropy increases with decreasing radius in the equatorial plane, and increases from the equator to the poles at a given radius. The entropy distribution shows significant inhomogeneities in the form of tangled filaments. These can be explained in terms of convective motions in the unstably stratified medium. Indeed, the convective motions seen in this MHD model suggest that the flow is closely related to the CDAF discovered in viscous hydrodynamical simulations $(\S 1)$. In particular, Figure 8 shows that in Model A the time-averaged surfaces of constant specific entropy are closely aligned with those of specific angular momentum. This effect, noticed already for convective thick accretion disks by 
Bardeen (1970) and Paczyński \& Abramowicz (1982), and found in viscous hydrodynamic simulations by Igumenshchev et al. (1996) and Stone et al. (1999), indicates that the medium is marginally stable with respect to the Høiland criterion (Tassoul 1978). The polar regions of the flow, however, are filled with matter that has a very low density and high entropy and there is no convection there. We discuss the connection between Model A and the CDAF model in more detail in $§ 4.1$.

Figure 9 shows a snapshot of the flow streamlines in Model A projected on a meridional cross-section. Most of the flow is dominated by time-dependent vortices of different spatial scales. Matter accretes by gradually moving toward the black hole, passing through these vortices. Only in the narrow polar directions do the streamlines show regular inflow into the black hole. However, the accretion rate there is relatively small because of the low density. Figure 10 shows the time-averaged flow pattern, averaged over about three Keplerian rotation periods measured at $R_{\text {out }}$. The pattern consists of stationary large scale $(\sim R)$ meridional circulation cells. Thus, even the time-averaged flow shows deviations from a simple radial pattern.

We should emphasize that neither the snapshot in Fig. 9 nor the time-averaged flow pattern in Fig. 10 exhibits any tendency to produce a significant bipolar outflow of mass. In Fig. 10, there is an equatorial outflow inside a radius $R \simeq 10 R_{g}$. Other than this, there is no evidence for an outflow or a jet, in agreement with a general theoretical argument by Abramowicz, Lasota \& Igumenshchev (2000).

Figure 11 shows time-averaged radial profiles of selected gas properties on the equatorial plane. The upper left panel shows the density $\rho$. Leaving aside the spiky outer region near the injection radius and the innermost region near the black hole, we see that the density follows a roughly power-law behavior with radius, say over the range $R \simeq 10-100 R_{g}$. The upper right panel shows the specific angular momentum $\ell$. We see that the rotation is super-Keplerian over a small range of radius near the outer boundary, is sub-Keplerian over much of the intermediate region, and is nearly Keplerian near the black hole. The overall behaviour is similar to that found in global models of thick disks, slim disks and ADAFs (e.g., Jaroszyński et al. 1980; Abramowicz et al. 1988; Narayan, Kato \& Honma 1997; Chen, Abramowicz \& Lasota 1997). The super-Keplerian nature of $\ell$ near the injection point was discussed by Abramowicz, Igumenshchev, \& Lasota (1998). The lower left panel shows the radial velocity $v_{R}$, which is found to have a very non-regular behaviour. This is explained by the meridional circulation patterns seen in the time-averaged flow shown in Fig. 10. The spikes in the plot are due to changes in the sign of the velocity. The lower right panel shows the ratio $c_{s} / v_{K}$, which characterizes the relative thickness of the flow: $H / R \simeq c_{s} / v_{K}$. The ratio does not change much with radius. 
Over the interior of the flow, between about $10 R_{g}$ and $100 R_{g}$, we find approximately that $\rho \propto R^{-1}, c_{s} \propto R^{-0.5}$, and $\ell \propto \ell_{K} \sim R^{1 / 2}$. The radial velocity $v_{R}$ does not show a very clear power-law behavior.

Figure 12 shows the average total stress $T_{R \phi}$ in meridional cross-section:

$$
T_{R \phi}=\overline{\rho \delta v_{R} \delta v_{\phi}}-\overline{\rho \delta v_{A R} \delta v_{A \phi}} \equiv T_{R \phi}^{R}+T_{R \phi}^{m a g},
$$

where $T_{R \phi}^{R}$ is the Reynolds stress associated with fluid motions and $T_{R \phi}^{m a g}$ is the Maxwell stress associated with magnetic field fluctuations. In the definition of the latter, $\delta \mathbf{v}_{A} \equiv \delta \mathbf{B} / \sqrt{4 \pi \rho}$ represents the fluctuations in the Alfven speed due to field fluctuations. We find that $T_{R \phi}$ is nearly axisymmetric. Figure 12 shows that the sign of $T_{R \phi}$ behaves non-monotonically over much of the flow. Regions of negative $T_{R \phi}$ form regular structures which are extended in the vertical direction and are strongly correlated with the locations of circulation patterns in Fig. 10. In particular, there is an extended region of negative $T_{R \phi}$ at intermediate angles between the poles and the equator, where angular momentum is transported inward. Near the equator, however, the angular momentum is on average transported outward. We discuss the behavior of the stress in greater detail in $§ 4.1$.

Simulations on nested Cartesian grids can suffer from artificial perturbations in the flow at subgrid interfaces. These perturbations are due to the sudden change in the resolution across subgrids, which can introduce errors in interpolation. To check the influence of these errors in our simulations, we have calculated several test models on a spherical grid. These simulations showed good qualitative and quantitative agreement with models calculated on the nested Cartesian grid, indicating that any perturbations due to the presence of subgrids are weak. Although the spherical grid has important advantages on account of its simple and monotonic structure, we found that it is not practical to use it for the full range of simulations. This is because models on a spherical grid require significantly smaller time steps on account of the Courant condition near the poles (see the discussion at the beginning of $\S 3$ ) .

\subsection{Simulations with Poloidal Injected Field}

The previous section described simulations with a toroidal injected field. Here we describe analogous models with a poloidal field. In these simulations, we assumed that the injected gas has a vector potential with $A_{r}=A_{z}=0$ and a non-zero $A_{\phi}$. The range of $\beta_{0}$ that we have used varies from $10^{2}$ to $10^{4}$, which means that the injected field is always much below equipartition strength. We find that decreasing $\beta_{0}$ speeds up evolution of the model to the steady-state; however, as in the toroidal case, the final steady-state does not depend on $\beta_{0}$. We have simulated several low resolution models on a nested Cartesian grid, using $1-2$ 
subgrids. We refer to our representative model, calculated on 2 subgrids with $R_{\text {out }}=64 R_{g}$, as Model B. Because of the low resolution of the models, in the following we focus only on qualitative aspects of the evolution of the models.

The Keplerian differential rotation of the injected material stretches the poloidal magnetic lines in the azimuthal direction. This leads to a redistribution of angular momentum and spreading of the matter in the radial direction. A good fraction of the injected gas moves outward and leaves the computational domain. The rest moves inward and forms an accretion flow. At the beginning of the simulations, the magnetic field in the injection torus has zero net $B_{z}$. At later times, however, as the outflowing matter leaves the computational domain and carries away magnetic field of predominantly one sign of $B_{z}$, the inflowing matter drags magnetic field with the opposite sign of $B_{z}$ inward. As a result, the accretion flow acquires a non-zero net $B_{z}$.

We find that the evolution of the accretion flow goes through two distinct stages. First, there is a transient stage during which the gas in the injection torus spreads out and forms an accretion disk stretching down to the black hole. This transient stage is then followed by a quasi-steady state in which the magnetic field exerts a significant back-pressure on the accreting gas.

In the transient stage, the geometry of the flow is very similar to that described by Hawley, Balbus \& Stone (2001) in their 3D simulations of non-radiative MHD flows. The accreting gas forms a nearly Keplerian disk with a vertically extended low-density corona. The magnetic field has a simple dipole topology near the outer boundary, but tends to be complicated at smaller radii, with toroidal fields of opposite directions above and below the disk. The field experiences reconnection events which cause the matter in the equatorial region to be locally heated up. Due to the combined effects of reconnection heat, toroidal magnetic field pressure and the centrifugal mechanism (Blandford \& Payne 1982), a fraction of the disk matter is ejected in the vertical directions to feed the low density corona. The corona is mostly in dynamical equilibrium, but a small fraction of the matter in it forms a hot, low density, high velocity, bipolar outflow. The outflow carries a significant fraction of the liberated energy in the form of kinetic energy, heat and Poynting flux. As a result, the accretion disk, though quite hot, is not as hot or thick as in Model A.

With increasing time, the above transient phase is replaced by a steady state configuration with very different properties. The change occurs because the dipole magnetic field that is dragged inward by the accretion flow, accumulates in the vicinity of the black hole. Note that, while gas can fall into the black hole and disappear, open field lines cannot vanish. With time, the accumulated dipole field becomes quite strong and its influence begins to extend to large radii. When the magnetic energy density reaches equipartition with the 
thermal energy density of the disk, the magnetic pressure is strong enough to suppress accretion. After this time, matter can move toward the black hole only by 'diffusing' through the field, which it does by means of local interchanges followed by reconnection. The simulations show that the accreting matter becomes significantly sub-Keplerian and is no longer disk-like. Instead, the matter forms narrow and very slowly rotating streams, which radially penetrate the highly magnetized low density medium near the black hole.

With time, the region over which the accumulated field dominates increases in size until

it finally fills the entire computational domain. At this point, the model has reached a quasi-steady-state in which the net magnetic flux no longer changes significantly with time. Instead, any new magnetic field line which is injected with new matter is pushed out through the outer boundary by the magnetic pressure. Matter, however, can decouple from the field, 'diffuse' through the existing magnetic lines along the radial narrow streams mentioned above and fall into the black hole.

Figure 13 shows the distribution of density in Model B in this steady-state, after more than 10 orbits at $R_{\text {out }}$. Four streams are seen as an increase of density in the equatorial plane. The accretion velocity in the streams is significantly sub-sonic and sub-Alfvenic. The fact that there are four symmetric streams is because we have simulated only a $90^{\circ}$ wedge. A full $360^{\circ}$ simulation is likely to find a different number of streams. Nevertheless, we believe that the basic result of this calculation, namely that accretion becomes highly non-axisymmetric, is robust. The topology of the magnetic lines in meridional cross-section is shown in Fig. 14. We see that the magnetic field is dominated by a strong dipolar component. The magnetic $\beta \sim 10^{-3}-10^{-4}$ in the regions away from the streams, and $\sim 1$ within the streams. Surfaces of constant total pressure $\left(P_{g}+P_{m}\right)$ are nearly spherical. Figure 15 shows the structure of the magnetic field projected on the equatorial plane. The structure is complicated and time-dependent, reflecting the irregular character of field dissipation in the dense accreting streams and low density coronal medium.

\section{DISCUSSION}

The results described in $\S 3$ show that the nature of a radiatively inefficient MHD accretion flow is very different depending on whether the injected material is dominated by toroidal or poloidal fields. In $\S \S 4.1$ and 4.2 below we compare the results we have obtained for these two limiting cases to our previous work on viscous hydrodynamic and MHD flows. Then, in $\S 4.3$, we compare our results to simulations done by other groups, and in $\S 4.4$, we discuss applicability of the results to real accretion flows. 


\subsection{Toroidal Field Injection}

Model A, which involves the injection of matter with a toroidal field configuration, has a number of distinct features (§3.1). Field amplification and reconnection leads to a significant level of heating in many localized regions spread all over the flow. The heating causes the average entropy to increase as a function of decreasing radius, as well as to increase from the equator to the pole (Fig. 7). Since the gradient of the entropy tends to be unstable (by the Høiland criterion), convective motions are set up which lead to considerable turbulence in the medium. We see evidence for this turbulence in all the diagnostics we have considered: density (Fig. 1), pressure (Fig. 2), magnetic field (Fig. 4, 5), plasma $\beta$ (Fig. 6), velocity streamlines (Fig. 9, 10). The well-developed turbulent eddies ensure that fluid elements cannot accrete smoothly down to the black hole from large radius, but rather must random walk through a series of eddies. This has the effect of suppressing the mass accretion rate onto the black hole.

Convection in radiatively inefficient accretion flows, or ADAFs, has been discussed for several years, mostly in the context of viscous hydrodynamics. The possibility of convection in ADAFs was emphasized by Narayan \& Yi (1994, 1995ab), but the surprisingly strong effect that convection has on the flow was not appreciated until numerical simulations were carried out by Igumenshchev \& Abramowicz (1999) and Stone et al. (1999). Igumenshchev \& Abramowicz (2000) found that convection is most important when the viscosity is weak, specifically when the viscosity parameter $\alpha$ is less than about 0.1 . This and other studies also showed that convection has the effect of suppressing the mass accretion rate onto the black hole.

Using the hydrodynamic simulations as a guide, Narayan et al. (2000) and Quataert \& Gruzinov (2000) developed an analytical model describing a new form of radiatively inefficient accretion called CDAF. The model has two important features.

First, it postulates that convection transports angular momentum inward rather than outward; that is, the Reynolds stress, $T_{R \phi}^{R}$, associated with the fluid fluctuations is negative. The viscous stress is, however, always outward, i.e., $T_{R \phi}^{v i s c}>0$. Thus, there is a competition between convection and viscosity. In fact, the two fluxes almost cancel each other in a CDAF so that the net angular momentum flux is much smaller than either of the fluxes individually: $0<T_{R \phi}^{R}+T_{R \phi}^{v i s c} \ll\left|T_{R \phi}^{R}\right|,\left|T_{R \phi}^{v i s c}\right|$.

Second, convection drives an outward energy flux $F_{c}$, which dominates over other forms of energy transport. As a result, the flow is driven to a state of constant convective luminosity with radius, i.e., $4 \pi R^{2} F_{c}=$ constant. Narayan et al. (2000) and Quataert \& Gruzinov (2000) showed that this immediately implies that the density should scale as $\rho \sim R^{-1 / 2}$. 
Both effects cause the mass accretion rate to be reduced. Note that the first of the two effects is specific to rotating flows, whereas the second can occur in either rotating or non-rotating flows. Indeed, Igumenshchev \& Narayan (2002) showed that spherical accretion of a non-rotating magnetized plasma leads to strong convection, which causes many of the effects seen in rotating CDAFs: constant convective luminosity, density varying as $R^{-1 / 2}$, and suppressed mass accretion.

The presence of well-developed convection in Model A suggests that this rotating MHD model may be similar to a CDAF. For instance, Model A has a relative disk thickness, $H / R \simeq 0.5$, a flattened density profile, and nearly Keplerian rotation, all of which make it similar to the CDAF model shown for instance in Fig. 17 of Igumenshchev \& Abramowicz (2000). There is, however, a controversy in the literature on whether or not an MHD flow can behave like a CDAF. Balbus \& Hawley (2002), based on linear analysis, claimed that it is highly unlikely that there could be modes in an MHD accretion flow that transport angular momentum inward (see also Christodoulou, Contopoulos \& Kazanas 2003, who come to a similar conclusion), whereas Narayan et al. (2002) argued that convective modes in an MHD medium can transport angular momentum inward provided the mode wavelength $\lambda$ is long enough; specifically, they required $\lambda / H \gg \beta^{-1 / 2}$.

Figure 12 shows the distribution of total stress $T_{R \phi}$ in the meridional plane of Model A. We see that the stress is both positive and negative in different regions, which makes it hard to identify in which direction angular momentum flows on average. Figure 16 shows separately the spherically averaged Reynolds stress $T_{R \phi}^{R}$ (filled dots) and Maxwell stress $T_{R \phi}^{\text {mag }}$ (open circles) as functions of the radius. Each quantity has been calculated over spherical shells of thickness equal to the inner radius. Thus, the average over a shell from $R$ to $2 R$ is plotted at the geometric mean radius $\sqrt{2} R$, the average from $2 R$ to $4 R$ is plotted at $2 \sqrt{2} R$, etc. In comparing the results to the idealized CDAF model, it should be noted that the Maxwell stress in an MHD medium plays the same role as the viscous stress $T_{R \phi}^{v i s c}$ in a viscous hydrodynamic flow.

Despite the coarse averaging procedure we have used, which corresponds to heavy smoothing, the results are unfortunately still noisy. This is a consequence of the fact that there are large-scale circulation patterns in the flow which introduce correspondingly largescale effects on the stress (e.g., Fig. 12). Nevertheless, some effects appear to be clear. First, we see that the Maxwell stress is always positive, i.e., the magnetic stress always moves angular momentum outward, just as viscosity does in the hydrodynamic case. Secondly, and more importantly, we see that over a good fraction of the interior of the flow (i.e., away from the boundaries), the Reynolds stress tends to be negative, i.e., the convective eddies move angular momentum inward. The sum of the two stresses is always positive, which means that 
angular momentum is moved outward on average. These features are qualitatively similar to what is seen in a hydrodynamic CDAF and suggest that MHD flows with toroidal field injection are analogous to the CDAF. One should keep in mind, however, that the numerical results are noisy. In addition, there is not a near cancellation of the two stresses, as expected in the idealized CDAF model. Rather, the Maxwell stress dominates over the Reynolds stress (as indeed expected by Balbus \& Hawley 2002). This may be the result of the relatively coarse resolution and small radial dynamic range of the present simulations. Another possible consequence of the net outward angular momentum flux is that an MHD CDAF might consist of two distinct parts: an almost non-rotating inner part and a quasi-Keplerian rotating outer part (Igumenshchev 2002).

As mentioned above, another feature of the CDAF model is that the density has a shallow radial dependence, $\rho \sim R^{-1 / 2}$, compared to the steeper dependence, $\rho \sim R^{-3 / 2}$, expected in the absence of convective angular momentum transport (Narayan \& Yi 1994). Fig. 11 shows that the density in Model A is indeed shallower than $R^{-3 / 2}$, which is consistent with the expectations of the CDAF model. However, the profile is not as shallow as the theoretically expected $R^{-1 / 2}$, but rather appears to vary as $\sim R^{-1}$. We note in this context that not all numerical simulations of CDAFs give $R^{-1 / 2}$. Igumenshchev \& Abramowicz (2000), for instance, found $\rho \sim R^{-0.5}$ for $\gamma=5 / 3$, but $\sim R^{-0.7}$ for $\gamma=4 / 3$.

One possible explanation for the discrepancy in the radial dependence of the density is that convection moves energy not only in the radial direction, as assumed in the simple one-dimensional CDAF model developed by Narayan et al. (2000) and Quataert \& Gruzinov (2000), but also in the vertical direction. Imagine dividing the flow into an equatorial thick 'disk' region where most of the accretion occurs and a polar 'corona' region. In the disk region, apart from convective energy transport in the radial direction, there could also be vertical leakage of energy into the corona. Such a vertical component of the convective flux has been seen in viscous hydrodynamical simulations (e.g. Abramowicz, Björnsson \& Igumenshchev 2000). Obviously, if there is a loss of energy from the disk, then the radial convective luminosity $R^{2} F_{c}$ within the disk will not be independent of radius, as assumed in the CDAF model, but will decrease with increasing radius. It is easy to see that this will cause the density profile to steepen relative to the pure CDAF profile.

To be more quantitative, let us consider the same form of the energy equation as in Narayan et al. (2000), but with an additional cooling term $Q^{-}$to describe the vertical loss of energy,

$$
\rho v T \frac{d s}{d R}+\frac{1}{R^{2}} \frac{d}{d R}\left(R^{2} F_{c}\right)=Q^{+}-Q^{-},
$$

where $s$ is the specific entropy, $T$ is the temperature, $F_{c}$ is the outward convective energy flux through the disk and $Q^{+}$is the energy dissipation rate per unit volume. In the CDAF 
solution, one neglects all the terms except the second one on the left and thereby finds that the convective luminosity $4 \pi R^{2} F_{c}$ is independent of radius. For the present case, let us retain the cooling term and write

$$
\frac{1}{R^{2}} \frac{d}{d R}\left(R^{2} F_{c}\right)=-Q^{-},
$$

and let us specify $Q^{-}$by assuming that a fraction $\xi$ of the outward flux $F_{c}$ escapes in the vertical direction:

$$
Q^{-}=\xi \frac{F_{c}}{H} .
$$

In radiatively inefficient accretion flows the temperature is close to virial, so the outward convective flux can be estimated to be

$$
F_{c} \propto \rho v_{K}^{3} \propto \rho R^{-3 / 2} .
$$

Substituting the estimate (13) into equation (11), we find that $\rho$ varies as $R^{-a}$, where

$$
a=\frac{1}{2}+\frac{\xi}{H / R} .
$$

Equation (14) shows that, in the presence of an extra source of cooling as represented by a value of $\xi>0$, the radial density profile becomes steeper than the canonical $R^{-1 / 2}$ of the CDAF model. Typically, we have $H / R \approx 0.5$. Thus, in order to explain the slope of $a \sim 1$ found in Model A, we require $\xi \sim 1 / 4$. Thus, about $25 \%$ of the local convective energy flux in the disk must escape into the corona.

It should be noted that, despite the modification described above, which allows for vertical loss of energy and a range of power-law indices $a$ for the density, this model is still basically a CDAF, since it is convection that causes the index $a$ to fall below $3 / 2$. Another model that has been discussed in the literature (Blandford \& Begelman 1999) invokes heavy mass loss in an outflow as a result of a positive Bernoulli constant in the accreting gas (Narayan \& Yi 1994, 1995ab). This model again leads to a range of values of $a$, but it is very different in spirit from the CDAF. In the mass outflow model, the mass accretion rate in the disk is large at large radii and decreases as one moves in. The excess mass is lost from the system in a powerful mass outflow. Model A exhibits significant mass outflow only in the outermost region, near the radius where mass is initially injected into the simulation. Further in, there is practically no mass lost from the system. Rather, the gas here participates in convective eddies. Any fluid element that temporarily moves outward as a result of buoyancy turns around after going some distance and flows back toward the center. In this sense, Model A is closer in spirit to a CDAF than the mass outflow model of Blandford \& Begelman (1999). 


\subsection{Poloidal Field Injection}

Model B, in which the injected matter has a poloidal magnetic field, behaves completely differently from Model A. In an initial, transient stage, the gas in this model forms a relatively flattened disk, with a hot corona and a low-density, high-velocity, bipolar outflow. There is some qualitative analogy between this stage of the flow and what has been seen in viscous hydrodynamic simulations with large viscosity parameter $\alpha \sim 0.3-1$ (Igumenshchev \& Abramowicz 2000). Those flows too have powerful unbound outflows. However, the analogy is not perfect. For example, the high- $\alpha$ hydro flows have a large accretion velocity and very sub-Keplerian rotation, whereas the MHD Model B described here has a small accretion velocity and nearly Keplerian rotation.

After the above transient phase, Model B settles down to a completely different configuration which is characterized by a strong dipolar field that fills the entire computational domain. The field builds up to this configuration during the transient phase as the accreting gas advects in magnetic field with the same sign of mean $B_{z}$. The accumulated field imposes strong constraints on the infalling gas. Once the steady state has been reached, any injected magnetic field is carried away from the computational domain by a fraction of the injected gas, while the rest of the gas, minus field, accretes onto the black hole via long slowly-rotating nearly-radial streams.

The reason for the buildup of magnetic field can be traced to the particular prescription we have used for the injected field, in which the vector potential $\mathbf{A}$ is assumed to be independent of time. Because of this, we continuously inject matter with the same sense of

poloidal field. The gas near the inner radius of the torus always has one sign of $B_{z}$ and it is this gas that accretes onto the black hole, causing a steady buildup of the magnetic flux. The gas near the outer radius of the injection torus escapes from the computational domain, carrying away flux of the opposite sign.

This explains why we find such a profound difference between simulations with toroidal field injection (Model A) and poloidal field injection (Model B). In the former case, the injected gas has no net magnetic flux. Therefore, even though part of the gas accretes and part escapes, the part that accretes does not carry a net flux and there is no opportunity for the flux to build up near the black hole.

Somewhat analogous behavior to that described above for Model B has been seen in spherical MHD accretion. Igumenshchev \& Narayan (2002) simulated Bondi accretion from an external medium in which the external gas had a uniform magnetic field. They found that, as the gas flows in, the field around the black hole builds up, causing a back-reaction on the accreting gas. This is one of the reasons (though the main reason is convection) for 
causing the accretion rate to fall. The main difference between the Bondi simulation and Model B described here is the geometry of the injected mass. In the Bondi problem, the mass comes in spherically, whereas in Model B, the mass is injected in a torus with a disklike geometry. With a disk-like source, there is a large solid angle of empty space through which a low density outflow can carry away energy and angular momentum. However, when the incoming mass has spherical symmetry, any gas that attempts to carry away energy is stopped by incoming gas. As a result, it is more efficient for the gas to transport energy via convective motions and this is what is seen in the Bondi simulation. As the magnetic field at the center builds up, however, the spherical symmetry is broken and the accreting matter in the inner regions takes the form of a doughnut (see Igumenshchev \& Narayan 2002).

\subsection{Comparison with Previous MHD Simulations}

Several groups have recently published results of 2D and 3D MHD simulations of radiatively inefficient accretion flows. Machida et al. (2001) studied the evolution of a rotating magnetized torus in which the magnetic field initially is purely toroidal. During the evolution, the torus forms a turbulent accretion flow with a flattened radial density profile. The authors state that their flow is similar to a CDAF, and indeed their simulation has many points of resemblance to our Model A. A detailed quantitative comparison of the two models is, however, difficult since their simulation extends over a limited range of radius, so that there are large boundary effects.

The study cited above, and several others, have either not used explicit resistive terms in any of the MHD equations, or used resistivity only in the induction equation (4) but without accounting for resistive dissipation in the energy equation (the last term on the right in eq.[3]). These simplifications could cause serious non-conservation of energy: one has a reduction of magnetic energy via numerical reconnection or resistive dissipation, but the reduction is not compensated for by a corresponding increase in the thermal energy (see $\S 2.1$. Non-conservation of energy in magnetic reconnection will lead to a suppression of thermal convection (Igumenshchev \& Narayan 2002).

Stone \& Pringle (2001), Hawley et al. (2001) and Hawley \& Balbus (2002) have presented simulations of MHD nonradiative accretion flows, starting from an initial torus of matter with a poloidal magnetic field. Their simulations are very relevant to our Model B. The initial configuration of the field in the simulations is such that the inner half of the torus (the parts of the torus inside the radius of maximum pressure) has vertical magnetic flux of one sign, while the outer half has magnetic flux of the opposite sign. Initially, the two halves exactly cancel each other, and so the total vertical magnetic flux integrated over the com- 
putational domain is zero. However, with time, the torus spreads in the radial direction, the inner half moving toward the black hole and the outer half moving outward. This spreading causes the regions with opposite signs of magnetic flux to move apart. Therefore, as in our Model B, the inner part of the torus forms an accretion flow which carries inward a net vertical magnetic flux of one sign (the same sign as the inner half of the initial torus). Flux of the opposite sign moves outward and leaves the computational domain on the outside, again exactly as in Model B.

The above authors found that the inflowing matter forms a rotationally supported disklike accretion flow sandwiched between low-density coronal regions. The coronal regions form bipolar outflows. The net magnetic field in the innermost regions of their model has a bipolar structure with non-zero net vertical flux (see Figs 4 and 5 of Stone \& Pringle 2001). These features are similar to what we see in our Model B during its initial transient stage. However, there are two important details in which the models of Hawley \& Balbus (2002) differ from our simulations. They report finding a small hot torus near the marginally stable orbit and a magnetically-confined jet near the rotation axis. These are not present in Model B. An even more important difference is that Hawley et al. (2001) and Hawley \& Balbus (2002) do not see any accumulation of magnetic field in the vicinity of the black hole. Consequently, their simulations do not exhibit the kind of steady state configuration we have described in $§ 3.2$ in which the field dominates the dynamics and gas is able to accrete only via narrow radial streams.

We believe that this discrepancy is the result of using different inner boundary conditions. We use a spherical inner boundary condition centered on the black hole (see §2.2). Thus, the black hole is the only absorbing entity, and the $z$-axis away from the black hole is a non-absorbing regular region of the flow. In contrast, Hawley and collaborators use a cylindrical inner boundary condition, in which the region within $1.5 R_{g}$ cylindrical radii of the $z$-axis is removed from the computational domain and absorbing boundary conditions are applied along this entire excised region. While the two boundary conditions give similar results for hydrodynamic simulations, they cause significant differences for MHD flows.

Figure 17 schematically shows the difference between the two boundary conditions. When magnetized gas flows in, the net magnetic flux in the accretion flow is conserved if the spherical boundary condition is used. Thus, matter falls into the black hole, but open field lines do not disappear. In contrast, with the cylindrical boundary condition, the component of the magnetic field parallel to the axis of the cylinder gets absorbed, and the simulation does not conserve the net magnetic flux. Whether or not a magnetic field line disappears entirly from the grid depends on the velocity streamlines of the accreting gas. For instance, under certain special conditions a field line may remain stuck in the configuration 2 shown 
in the left panel of Fig. 17. However, even in this case, the region in the vicinity of the black hole loses the magnetic pressure associated with the advected field line and will behave differently compared to the spherical boundary case. This is a serious problem, especially for accretion flows such as Model B. In our simulation of Model B, the accumulated field remains in the computational volume and has a profound effect on the time evolution of the accretion flow. With the cylindrical boundary condition, however, the field never has a chance to accumulate since it keeps disappearing at the axis. Therefore, one obtains an incorrect description (in our view) of MHD processes in the vicinity of the black hole.

The cylindrical inner boundary condition is convenient and allows one to avoid certain technical problems near the rotation axis in 3D MHD simulations. Perhaps for this reason, a number of recent numerical simulations of MHD accretion flows around black holes have employed this boundary condition (e.g. Hawley 2000, 2001; Hawley \& Krolik 2001, 2002; Armitage, Reynolds, \& Chiang 2001; Krolik \& Hawley 2002). The effect of the inconsistency pointed out above on these simulations needs to be investigated.

2D numerical simulations do not usually suffer from this problem, so one would expect the magnetic flux accumulation that we find in Model B to be apparent in such work. The simulations of Stone \& Pringle (1999) are interesting in this respect. Although they start with an initial torus with a fixed amount of matter rather than a torus with continuously injected mass as in our work, nevertheless, at late times when much of the mass has accreted, their simulation ought to exhibit the magnetic field accumulation at the center that we find. Unfortunately, it appears that their calculations were not run long enough. There is some indication in Fig. 6 of their paper that the magnetic pressure is becoming dynamically important at small radii. This is similar to the effect that we see in our Model B, except that our effect is much larger and more dramatic.

Recently, Casse \& Keppens (2002) published results of 2D numerical simulations of a magnetized accretion disk with a polytropic equation of state. The authors assumed continuous injection of matter at the outer boundary, which makes their work closer to our simulation compared to the Stone \& Pringle work. Unfortunately, they have evolved their model for a very short time, less than an orbital period at the injection radius. Their results are consistent with those of Hawley and collaborators and also with our results on Model B during the early transient phase. However, since they have not run their model long enough, they are not in a position to confirm the late-time field-dominated steady state that we find.

After the present paper was submitted to the journal, Proga \& Begelman (2003) posted a paper describing 2D MHD simulations of slowly-rotating radiatively inefficient gas on a black hole. They introduce a new magnetic field topology for this problem, namely a split monopole configuration. While there are interesting points in their work, we note that the 
split monopole field is rather artificial - by construction, it always has a fixed magnetic flux! Thus, there is no possibility for the field near the black hole to build up over time.

Since the unusual steady-state that we find for Model B is the most surprising (and possibly controversial) result of the present paper, we take this opportunity to summarize the situation:

1. In the present work, we have run a 3D MHD simulation (Model B) for a long time, using a poloidal field configuration in the injected gas such that the portion of the material that accretes always carries in the same sign of $B_{z}$. We find that the magnetic flux accumulates, initially near the black hole and then farther out, and the field becomes dynamically dominant. At late time, mass is able to accrete only via narrow streams, in a highly non-axisymmetric manner.

2. Igumenshchev \& Narayan (2002) carried out 3D MHD simulations of spherical accretion of gas with a uniform $B_{z}$. Their simulation showed evidence for field accumulation at the center. If the simulation were run for a longer time, we predict that the field would dominate the entire computational domain, and would have effects similar to what we see in Model B.

3. Hawley et al. (2001) and Hawley \& Balbus (2002) have carried out 3D MHD simulations of rotating flows, but do not see any evidence for field accumulation at the center. We argue that their boundary condition (see Fig. 17) does not conserve magnetic flux. Therefore, in our view, their simulations will not exhibit field accumulation near the black hole even if run for a very long time.

4. Stone \& Pringle (1999) have run 2D MHD simulations, starting from an initial magnetized torus. There is a slight hint that their final state has some field accumulation at the center. If their simulation were run for a longer time, such that most of the gas either accretes or is ejected from the computational domain, then we believe that the final configuration would correspond to the field-dominant state found in Model B. There would be one difference, however. Since the Stone \& Pringle simulation is in 2D, it cannot show the kind of nonaxisymmetric accretion via streams that we see in Model B.

5. Casse \& Keppens (2002) have carried out 2D simulations with continuous mass injection in a torus. If these simulations are run for a long time, they should resemble Model B. However, being 2D, they again will not find the accretion streams that we see in Model B.

6. Finally, Proga \& Begelman (2003) have done 2D simulations with a very special magnetic field geometry (split-monopole) that forces the magnetic flux to remain constant in time. This simulation will not reproduce the flux accumulation that we see in Model B regardless of how long it is run. 


\subsection{Is Model B Relevant for Real Accretion Flows?}

Leaving aside the conflicting results from different numerical simulations, which we have attempted to sort out in the previous subsection, the physical concepts behind the long-term evolution of Model B appear to be sound. Namely, if gas accretes on a black hole with magnetic field of a single sign of $B_{z}$, then magnetic flux will accumulate around the hole and will, over time, become dynamically important. The field will then disrupt the accretion flow and will cause the gas to flow in via non-axisymmetric streams or blobs, which will almost certainly have observational consequences. But how relevant are our results for real accretion flows?

The particular geometry of injected matter that we have considered in Model B is somewhat artificial. We have assumed that the injection occurs in a narrow torus, with a poloidal field configuration that is so designed that the inflowing and outflowing gas have opposite signs of $B_{z}$. This particular geometry is unlikely to occur in nature. However, the final steady state that we see in Model B does not depend on the precise geometry. All that is required is that the accreting gas should have a net $B_{z}$ of a single sign (positive or negative) over an extended period of time. This is by no means unlikely.

In a real accretion flow we imagine that $B_{z}$ will fluctuate with time. If the fluctuations are slow so that the sign of $B_{z}$ remains the same for much longer than the accretion time, then the flow ought to resemble what we see in Model B. There should be a transient period during which the gas would form a disk and would have an energetic outflow, followed by a long period of time during which the field would be dominant over a central volume near the black hole and accretion would be restricted to narrow streams. When the sign of the field finally reverses (assuming it does — see below), there will again be a transient disk-like phase with ejection in an outflow, etc., and the cycle will repeat. During the transient stages, one would expect to observe flare activity due to the annihilation of oppositely directed poloidal fields. Note that in this scenario the flare activity could be very powerful and easily detectable in observations, because stored energy of dissipated magnetic field is comparable to the binding energy of accretion matter. If the poloidal field reverses its direction frequently, then we imagine that the accretion flow will resemble the initial transient phase of Model B at all times. Both cases could be investigated by repeating our simulations with a time-varying $A_{\phi}$ in the injected gas.

The long-term evolution of the magnetic field near the black hole depends on the statistics of the magnetic field in the externally supplied gas. If the $\Delta B_{z}$ that is added with each new piece of matter is completely uncorrelated with previous injections, then the accreted magnetic flux would random-walk in time, and would on average increase as $t^{1 / 2}$. Alternatively, if the accreting matter is supplied by an external medium that experiences MHD 
turbulence with a large outer scale, then one expects the flux to grow to a large value and to remain at that value for a long time (as long as it takes gas to accrete from a radius of order the outer scale). If, however, the turbulent spectrum is dominated by small-scale field, then the flux would not grow very much and the vertical field component would always be sub-equipartition. In this case one might expect the formation of a CDAF-type flow as in Model A. In the case of accretion in binary stars, the statistics of the magnetic field in the outer envelope of the mass-donor star will determine how much field accumulates around the primary. This is poorly understood.

Note that, in most accretion flows, we expect the magnetic field strength in the disk to be amplified via the magnetorotational instability, but the additional field so created will on average have zero $B_{z}$. Therefore, it is only the original $B_{z}$ supplied with the gas that is relevant for determining how much flux will accumulate around the accreting star.

The very unusual effects we have seen in Model B, viz., dynamically dominant magnetic field and accretion via narrow slow-rotating streams, may not even be restricted to radiatively inefficient accretion flows. A similar buildup of field and corresponding modification of gas dynamics could occur also in radiatively efficient accretion flows.

High-energy observations frequently suggest the simultaneous presence of hot nearly relativistic matter and 'cold' quasi-thermal gas in the inner regions of active galactic nuclei (AGN) and black hole X-ray binaries. Magnetic fields are believed to be a common ingredient in these systems, and it has been proposed that the field may effectively confine or otherwise couple to clumpy cold gas (Krolik 1998; Celotti \& Rees 1999). Model B exhibits some of the features postulated in these proposals. Accretion occurs mostly as relatively cold gas, but the binding energy of this gas is released through magnetic reconnection and a large fraction of the heat goes into the hot phase. $\mathrm{X} / \gamma$-rays radiated by reconnecting magnetic flares in the hot phase, and reprocessed optical-UV radiation from the cold phase, may explain some of the spectral signatures observed in AGN and X-ray binaries.

\section{SUMMARY}

We have described in this paper global time-dependent three-dimensional MHD simulations of radiatively inefficient accretion flows onto black holes. The simulations are done within the framework of a pseudo-Newtonian potential to mimic relativistic effects near the black hole. The flows extend from an inner radius $R_{\text {in }}=2 R_{g}$ to an outer radius $R_{\text {out }}$, which in different models ranges from $16 R_{g}$ to $512 R_{g}$. Magnetized matter is continuously injected into the computational domain within a torus whose major radius is slightly less than $R_{\text {out }}$ 
and whose minor radius is about one grid cell. The material starts off with Keplerian rotation and no initial radial velocity. However, matter spreads under the influence of magnetic torques, and part of the injected matter accretes onto the black hole while the rest escapes from the computational box. The following is a summary of the main results.

1. We have studied two limiting cases for the geometry of the injected magnetic field: pure toroidal field and pure poloidal field. We find that the results are completely different in the two cases.

2. When the injected field is primarily toroidal, which corresponds to the simulation designated Model A (see §3.1), the accreting gas forms a nearly axisymmetric, quasi-stationary, geometrically thick disk with little mass outflow in a wind or a jet.

3. The magnetic field achieves a saturation level with a plasma $\beta \sim 10^{2}$. In this saturated state, field amplification by radial convergence and dynamo action is balanced by field dissipation through reconnection.

4. There is considerable convective activity in the flow, driven by an unstable entropy gradient that results from energy dissipation via field reconnection.

5. There is a close analogy between our MHD Model A and the CDAF model that was developed to explain viscous hydrodynamic simulations of radiatively inefficient accretion flows. In particular, there is evidence that the Reynolds stress in the interior of the flow (away from the boundaries) moves angular momentum inward rather than outward.

6. The radial density profile is relatively flat, $\rho \sim R^{-1}$, but not as flat as the $\rho \sim R^{-1 / 2}$ predicted by the CDAF model. We suggest that the difference is because of the vertical leakage of convective energy flux from the thick disk (see $§ 4.1$ ).

7. These results on the toroidal field case are consistent with some previously reported work in the literature, but our simulations have a larger dynamic range and have been run for a longer time.

8. When the injected field is primarily poloidal, which corresponds to the simulation designated Model B (see §3.2), the accretion flow goes through two distinct phases.

9. In an initial transient phase, the accreting gas forms a relatively flattened axisymmetric disk with a hot corona and a bipolar outflow. This stage is similar to results found by other groups.

10. With time, as the accreting gas continues to bring in magnetic flux, the magnetic field builds up around the black hole and farther out. The strong field disrupts the axisymmetry of the disk, leading to a completely different steady state flow configuration. 
11. In steady state, there is a two-phase medium. Most of the volume is filled with a strong dipolar magnetic field with $\beta \ll 1$, from which a thermal wind flows out along the open magnetic lines. Accretion occurs mainly via narrow slowly-rotating radial streams, with $\beta \sim 1$, which 'diffuse' through the field.

We thank Eliot Quataert and the referee, Jim Stone, for many helpful comments on the manuscript. IVI was supported by the U.S. Department of Energy (DOE) Office of Inertial Confinement Fusion under Cooperative Agreement No. DE-FC03-92SF19460, the University of Rochester, the New York State Energy Research and Development Authority, and RFBR grant 00-02-16135. RN was supported by NSF grant AST-9820686. 


\section{REFERENCES}

Abramowicz, M. A., Czerny, B., Lasota, J.-P., \& Szuszkiewicz, E. 1988, ApJ, 332, 646

Abramowicz, M. A., Björnsson, G., \& Igumenshchev, I. V. 2000, PASJ, 52, 295

Abramowicz, M. A., Chen, X.-M., Kato, S., Lasota, J.-P., \& Regev, O. 1995, ApJ, 438, L37

Abramowicz, M. A., Igumenshchev, I. V., \& Lasota, J.-P. 1998, MNRAS, 293, 443

Abramowicz, M. A., Lasota, J.-P., \& Igumenshchev I. V. 2000, MNRAS, 314, 775

Armitage, P. J., Reynolds, C. S., \& Chiang, J. 2001, ApJ, 548, 868

Balbus, S. A., \& Hawley, J. F. 1991, ApJ, 376, 214

Balbus, S. A., \& Hawley, J. F., 2002, ApJ, 573, 749

Bardeen, J. M. 1970, ApJ, 162, 71

Begelman, M. C., \& Meier, D. L. 1982, ApJ, 253, 873

Blandford, R. D., \& Payne, D. G. 1982, MNRAS, 199, 883

Blandford, R. D., \& Begelman, M. C. 1999, MNRAS, 303, L1

Cabral, B., \& Leedom, L. 1993, Computer graphics: Proceedings: Annual Conference Series 1993: SIGGRAPH 93 (New York: Association for Computing Machinery), 263

Casse, F., \& Keppens, R. 2002, ApJ, 581, 988

Celotti, A., \& Rees, M. J. 1999, MNRAS, 305, L41

Chen, X., Abramowicz, M., \& Lasota, J.-P., 1997, ApJ, 476, 61

Christodoulou, D. M., Contopoulos, J., \& Kazanas, D. 2003, ApJ, 586, 372

Colella, P., \& Woodward, P. R. 1984, J. Comput. Phys., 54, 174

Gilham, S. 1981, MNRAS, 195, 755

Hawley, J. F. \& Balbus, S. A., 2002, ApJ, 573, 738

Hawley, J. F. 2000, ApJ, 528, 462

Hawley, J. F. 2001, ApJ, 554, 534 
Hawley, J. F., Balbus, S. A., \& Stone, J. M. 2001, ApJ, 554, L49

Hawley, J. F., \& Krolik, J. H. 2001, ApJ, 548, 348

Hawley, J. F., \& Krolik, J. H. 2002, ApJ, 566, 164

Ichimaru, S. 1977, ApJ, 214, 840

Igumenshchev, I. V., \& Abramowicz, M. A. 1999, MNRAS, 303, 309

Igumenshchev, I. V., \& Abramowicz, M. A. 2000, ApJS, 130, 463

Igumenshchev, I. V., Chen, X., \& Abramowicz, M. A. 1996, MNRAS, 278, 236

Igumenshchev, I. V., Abramowicz, M. A., \& Narayan, R. 2000, ApJ, 537, L27

Igumenshchev, I. V., \& Narayan, R. 2002, ApJ, 566, 137

Igumenshchev, I. V. 2002, ApJ, 577, L31

Jaroszyński, M., Abramowicz, M. A., \& Paczyński, B. 1980, Acta Astr., 30, 1

Krolik, J. H. 1998, ApJ, 498, L13

Krolik, J. H, \& Hawley, J. F. 2002, ApJ, 573, 754

Lasota, J.-P. 1996, in Physics of Accretion Disks : Advection, Radiation and Magnetic Fields, eds S. Kato, S. Inagaki, S. Mineshige, J. Fukue (Amsterdam: OPA), 85

Li, X.-L. 2003, ApJ, submitted (astro-ph/0212503)

Machida, M., Matsumoto, R., \& Mineshige, S. 2001, PASJ, 53, L1

McKinney, J. C., \& Gammie, C. F. 2002, ApJ, 573, 728

Narayan, R., Igumenshchev, I. V., \& Abramowicz, M. A. 2000, ApJ, 539, 798

Narayan, R., Kato, S., \& Honma, F. 1997, ApJ, 476, 49

Narayan, R., Mahadevan, R., \& Quataert, E. 1998, in Theory of Black Hole Accretion Discs, ed. M. A. Abramowicz, G. Björnsson, \& J. E. Pringle (Cambridge: Cambridge Univ. Press), p148

Narayan, R., \& Yi, I. 1994, ApJ, 428, L13

Narayan, R., \& Yi, I. 1995a, ApJ, 444, 231 
Narayan, R., \& Yi, I. 1995b, ApJ, 452, 710

Narayan, R., Quataert, E., Igumenshchev, I. V., \& Abramowicz, M. A. 2002, ApJ, 577, 295

Paczyński, B., \& Wiita, P. J. 1980, A\&A, 88, 23

Paczyński, B., \& Abramowicz, M. A. 1982, ApJ, 253, 897

Proga, D., \& Begelman, M. C. 2003, submitted in ApJ, preprint: astro-ph/0303093

Quataert, E., \& Gruzinov, A. 2000, ApJ, 539, 809

Rees, M. J., Phinney, E. S., Begelman, M. C., \& Blandford, R. D. 1982, Nat, 295, 17

Shvartsman, V. F. 1971, Soviet Astron., 15, 377

Stone, J. M., \& Norman, M. L. 1992, ApJS, 80, 791

Stone, J. M., Pringle, J. E., \& Begelman, M. C. 1999, MNRAS, 310, 1002

Stone, J. \& Pringle, J. E., 2001, MNRAS, 322, 461

Tassoul, J.-L., 1978, Theory of Rotating Stars (Princeton: Princeton University Press) 
Fig. 1.- Snapshot of the density distribution in Model A in the $x-z$ plane (meridional cross-section). The box shown has a size of $128 \times 128 R_{g}$, with the black hole at the center. Magnetized gas with a toroidal field is injected continuously within a torus at radius $\simeq 510 R_{g}$ (well outside the box shown here). The color bar on the side indicates the scale for $\log \rho$ (in arbitrary units). Note that the gas is concentrated toward the equatorial plane (the horizontal axis) and increases toward the black hole. The fluctuations in the density are caused by convective motions. The two vertical polar funnels are filled with low-density matter.

Fig. 2.- Snapshot of the pressure distribution in Model A in meridional cross-section (see Fig. 1 for details). The color bar gives the scale for $\log P$ (in arbitrary units). In the two vertical polar funnels, the pressure is very small as there is very little matter there.

Fig. 3.- Schematic evolution of a perturbed magnetic field line in a differentially-rotating accretion flow. The unperturbed velocity is assumed to correspond to a Keplerian rotation profile. (a), (b), (c), (d) show the geometry of a magnetic line as a function of time. (a) shows a small initial perturbation of the line; (b), (c) the perturbed segment of the line is stretched by the Keplerian shear, and a magnetic loop is formed; (d) the field line reconnects and the magnetic loop separates from the line, causing a net amplification of the field strength relative to (a).

Fig. 4.- Snapshot of magnetic lines in Model A in the $x-y$ plane (equatorial cross-section). The box shown has a size of $64 \times 64 R_{g}$, with the black hole at the center. The component of field lines parallel to the plane is shown. Note the large number of magnetic loops, stretched in the azimuthal direction by the Keplerian rotation. The origin of the loops is schematically explained in Fig. 3.

Fig. 5.- Snapshot of magnetic lines in Model A in meridional cross-section for a box of size $64 \times 64 R_{g}$. The component of field lines parallel to the plane is shown. Except for the polar regions, the magnetic field elsewhere has a highly tangled morphology. This is the result of convection.

Fig. 6. - Snapshot of the distribution of the plasma $\beta \equiv P_{g} / P_{m}$ in Model A in meridional cross-section for a box of size $128 \times 128 R_{g}$. The color bar gives the scale for $\log \beta$. A complicated pattern is seen in the distribution of $\beta$. Regions of large $\beta$, or weak magnetic field, correspond to regions of reconnection and dissipation of magnetic energy. In the polar regions, where magnetic field has a more regular structure (see Fig. 5), the distribution of $\beta$ is more homogeneous. 
Fig. 7. - Snapshot of the distribution of specific entropy $s$ in Model A in the meridional plane for a box of size $128 \times 128 R_{g}$. The color bar gives the scale for log $s$. Note the complicated filamentary structure in the equatorial region and in the intermediate region between the poles and the equator. This is the result of field dissipation and convection. The two vertical polar funnels are filled with high-entropy low-density matter.

Fig. 8.- Time-averaged distribution of specific entropy $s$ (left) and specific angular momentum $\ell$ (right) in Model A in meridional cross-section. The averaging has been done over about three Keplerian rotation periods measured at $R_{\text {out }}=256 R_{g}$. Comparing the two plots, one sees that lines of constant $s$ are closely aligned with those of constant $\ell$ over much of the volume, with the exception of the two funnels. This indicates that the accreting gas is marginally stable to convection according to the Høiland criterion. Some local features are evident in the distributions of $s$ and $\ell$ in the equatorial region. These are due to the circulation patterns shown in Fig. 10.

Fig. 9. - Snapshot of velocity streamlines in Model A in meridional cross-section, for a box of size $64 \times 64 R_{g}$. The component of streamlines parallel to the plane is shown. Except for the polar regions, the flow pattern consists of a number of vortices and eddies, the result of convective turbulence.

Fig. 10.- Same as in Fig. 9, but for the time-averaged flow. The averaging has been done over about three Keplerian rotation periods measured at $R_{\text {out }}=256 R_{g}$. Instead of the large number of eddies seen in Fig. 9, one now sees large-scale meridional circulation patterns which are quite symmetric with respect to the equatorial plane. In the polar regions and in the vicinity of the black hole the streamlines are directed inward. In the innermost region, inside $10 R_{g}$, outflows are observed, but only near the equatorial plane. 

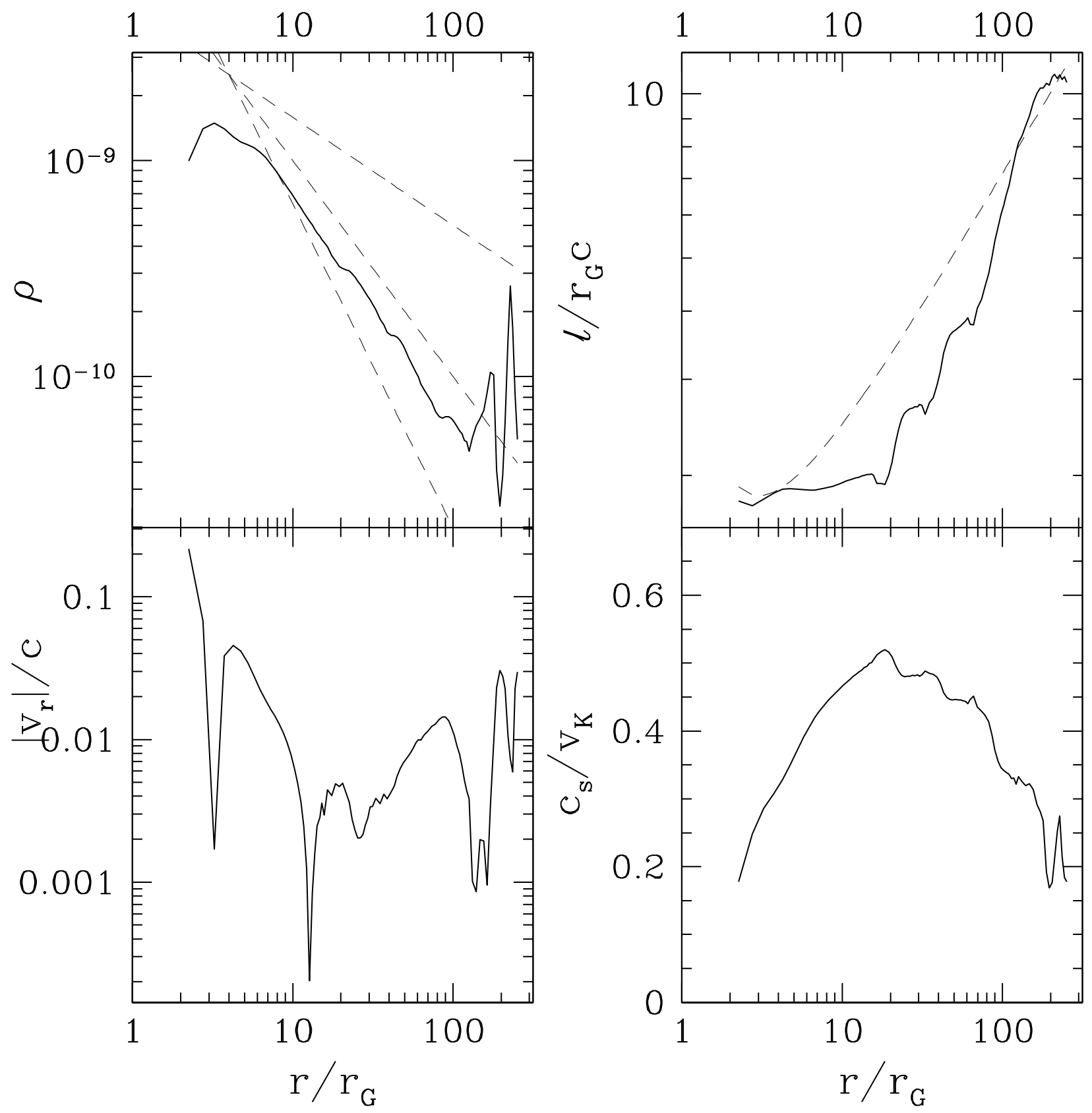

Fig. 11.- Radial structure of the flow in Model A on the equatorial plane. All plotted quantities - density $\rho$ (in arbitrary units), specific angular momentum $\ell$, radial velocity $v_{r}$, and sound speed velocity $c_{s}$ - have been averaged over about three Keplerian periods measured at $R_{\text {out }}=256 R_{g}$. The density distribution is shown together with three dashed lines, which represent power-laws $\propto R^{-a}$ with $a=1 / 2,1,3 / 2$. The specific angular momentum is shown along with a dashed line which represents the Keplerian distribution of $\ell$ for the pseudo-Newtonian potential (eq. 6) used in the simulation. 
Fig. 12.- Time-averaged distribution of the $R \phi$-component of the stress tensor, $T_{R \phi}$ (eq. [9]), in Model A in meridional cross-section for a box of size $128 \times 128 R_{g}$. The color bar gives the scale for the stress in units of the local time-averaged gas pressure. The black line separates regions of positive and negative stress (positive/negative stress correspond to outward/inward angular momentum flux, respectively). Note the correlation between the locations of negative and positive stresses and the time-averaged circulation pattern in Fig. 10 (remembering the different spatial scales in the two figures).

Fig. 13.- Density distribution in Model B in the $x-y$ plane (equatorial cross-section) at late times (steady state). The black hole is located at the center of a box of size $64 \times 64 R_{g}$. At this stage of the simulation, accretion occurs along almost radially directed streams moving in toward the black hole. One can see four symmetric streams in the image, which is the result of the simulation being done over a quarter of the domain, with azimuthally periodic boundary conditions (see $\S 3$ ).

Fig. 14.- Magnetic lines in Model B in the $x-z$ plane (meridional cross-section). The black hole is located at the center of a box of size $64 \times 64 R_{g}$. The component of field lines parallel to the plane is shown. The magnetic field clearly has a bipolar structure. The magnetic field is in rough equipartition with the accretion streams shown in Fig. 13.

Fig. 15. - Magnetic lines in Model B in equatorial cross-section for a box of size $64 \times 64 R_{g}$. Note the complicated structure of the field, which is loosely correlated with the positions of the accretion streams seen in Fig. 13. 


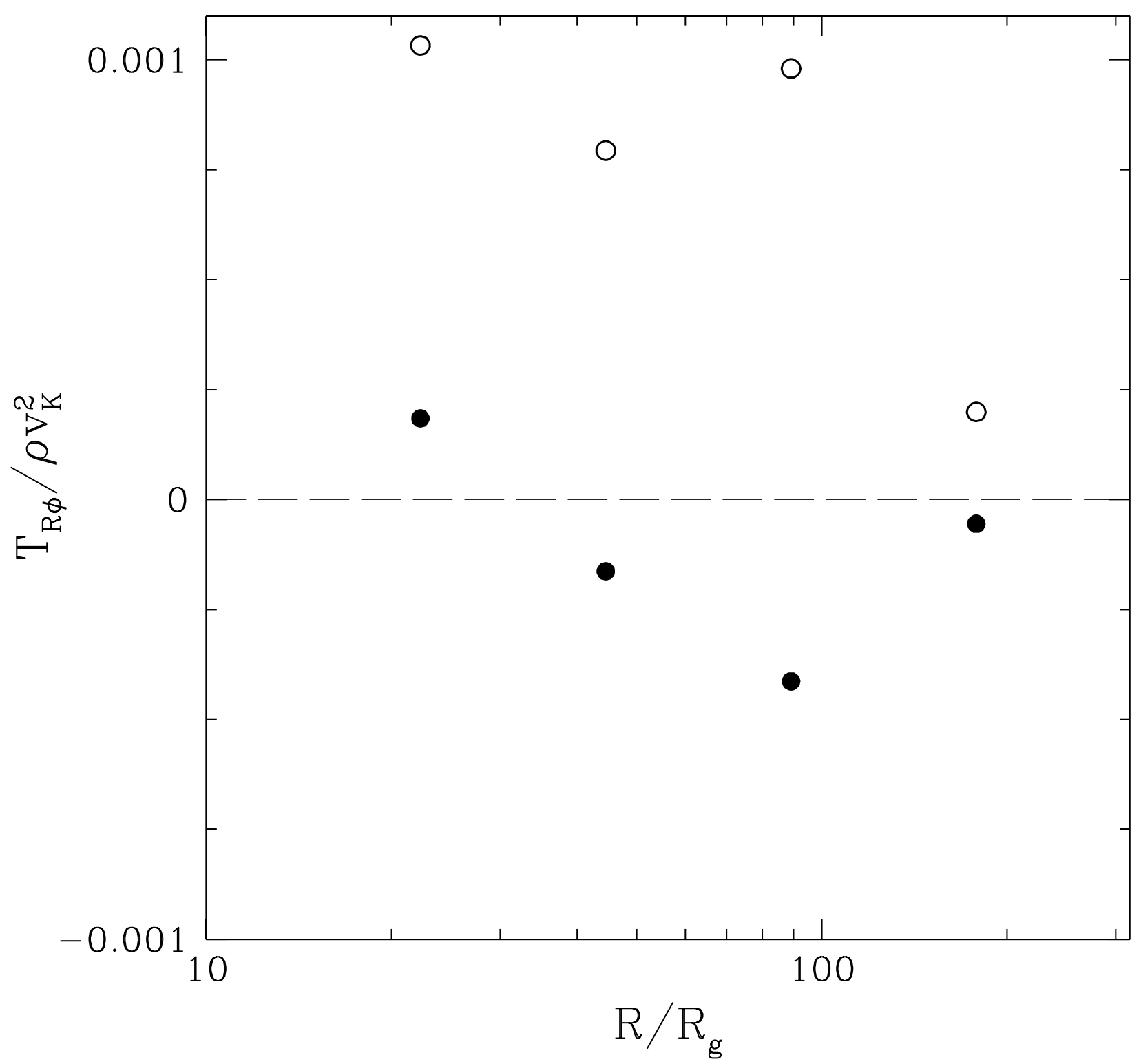

Fig. 16.- Radial distribution of Reynolds stress $T_{R \phi}^{R}$ (filled dots) and Maxwell stress $T_{R \phi}^{\text {mag }}$ (open circles) in Model A. The stresses have been averaged over spherical shells as described in the text. Note the negative sign of the Reynolds stress over a range of radius. In this region, convection transports angular momentum inward, in agreement with the fundamental postulate of the CDAF model. The positive sign of the Maxwell stress indicates that the magnetic field transports angular momentum outward. The total stress (sum of Reynolds and Maxwell stresses) is positive, as expected, so that on average the angular momentum is transported outward. 

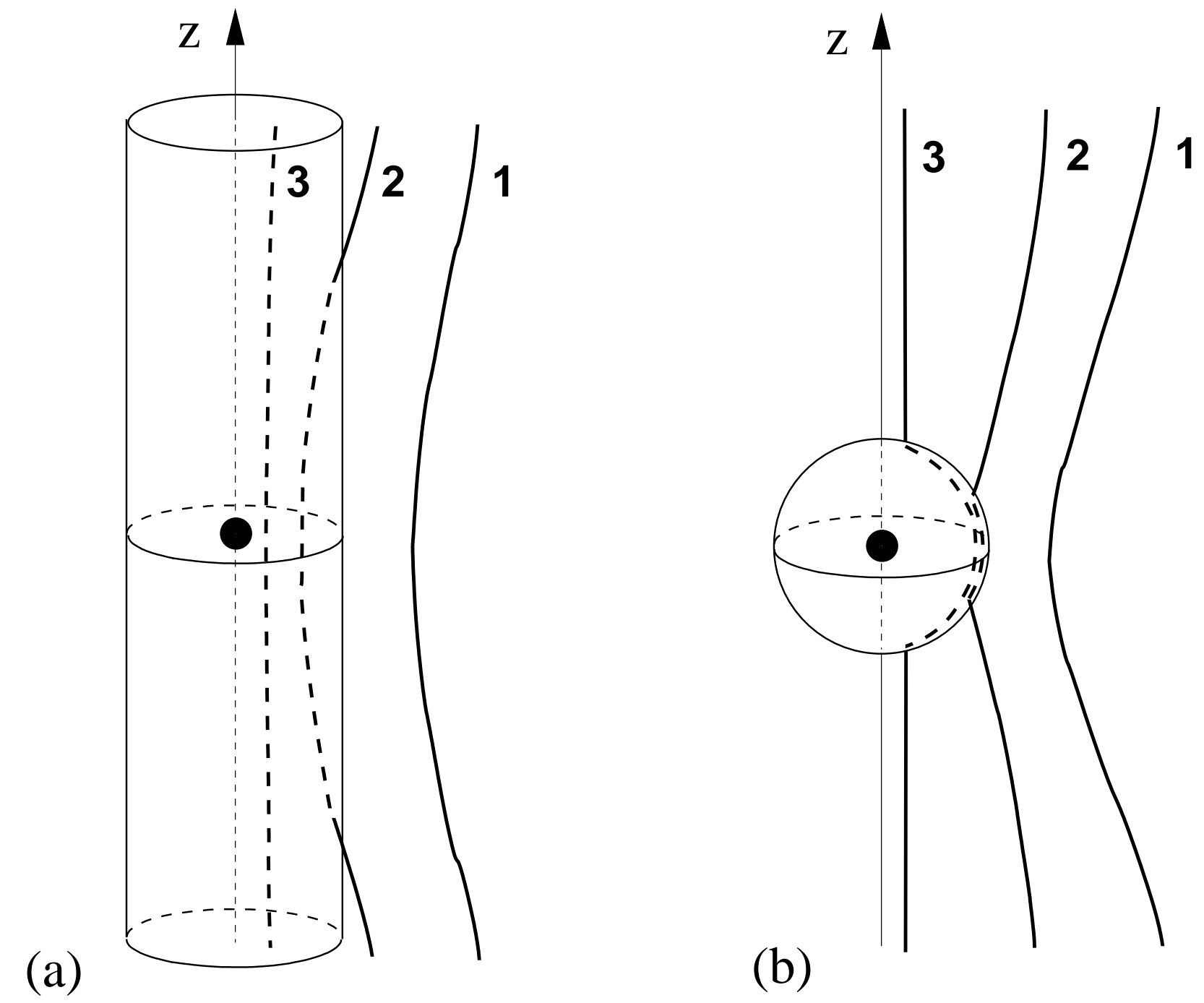

Fig. 17.- Cartoon depiction of the difference between two inner boundary conditions. Panel (a): shows the cylindrical boundary condition used by Hawley et al. (2001) and Hawley \& Balbus (2002). The cylinder goes through the whole computational domain, starting at the top outer boundary and ending at the bottom boundary. Panel (b): shows the spherical boundary condition used in the present study. The cylinder in panel (a) and the sphere in panel (b) indicate the locations of the absorbing boundaries in the two cases. Any matter or magnetic field that crosses the absorbing boundary stops contributing to the dynamics of the flow in the computational domain, thus mimicking the effect of gravitational capture of matter by the black hole. The bold lines 1, 2, 3 in each panel show the schematic time evolution of a frozen-in magnetic line as it is advected inward by the accretion flow. For simplicity, we do not consider the effects of rotation. In panel (a), the component of the magnetic field parallel to the $z$-axis is absorbed. Therefore, with time, a field line will either disappear entirely from the computational domain (e.g., line 3 which is entirely inside the absorbing cylinder) or in rare cases remain partially inside the grid (line 2). In either case, the simulation does not conserve the net magnetic flux in the vicinity of the black hole. In panel (b), on the other hand, open field lines do not disappear when the accreting matter falls into the black hole. Therefore, the net magnetic flux is conserved. This is shown by 
This figure "f1.jpg" is available in "jpg" format from: http://arxiv.org/ps/astro-ph/0301402v2 
This figure "f2.jpg" is available in "jpg" format from: http://arxiv.org/ps/astro-ph/0301402v2 
This figure "f3.jpg" is available in "jpg" format from: http://arxiv.org/ps/astro-ph/0301402v2 
This figure "f4.jpg" is available in "jpg" format from: http://arxiv.org/ps/astro-ph/0301402v2 
This figure "f5.jpg" is available in "jpg" format from: http://arxiv.org/ps/astro-ph/0301402v2 
This figure "f6.jpg" is available in "jpg" format from: http://arxiv.org/ps/astro-ph/0301402v2 
This figure "f7.jpg" is available in "jpg" format from: http://arxiv.org/ps/astro-ph/0301402v2 
This figure "f8a.jpg" is available in "jpg" format from: http://arxiv.org/ps/astro-ph/0301402v2 
This figure "f8b.jpg" is available in "jpg" format from: http://arxiv.org/ps/astro-ph/0301402v2 
This figure "f9.jpg" is available in "jpg" format from: http://arxiv.org/ps/astro-ph/0301402v2 
This figure "f10.jpg" is available in "jpg" format from: http://arxiv.org/ps/astro-ph/0301402v2 
This figure "f12.jpg" is available in "jpg" format from: http://arxiv.org/ps/astro-ph/0301402v2 
This figure "f13.jpg" is available in "jpg" format from: http://arxiv.org/ps/astro-ph/0301402v2 
This figure "f14.jpg" is available in "jpg" format from: http://arxiv.org/ps/astro-ph/0301402v2 
This figure "f15.jpg" is available in "jpg" format from: http://arxiv.org/ps/astro-ph/0301402v2 